\title{
Halomethoxylation of monoterpenes using (dichloroiodo)benzene
}

\author{
Mehman S. Yusubov, ${ }^{\text {a,b, }}$ Larisa A. Drygunova, ${ }^{\text {b }}$ Alexey V. Tkachev, \\ and Viktor V. Zhdankin ${ }^{{ }^{*}}$ \\ ${ }^{a}$ The Siberian Medical State University, 2 Moskovsky trakt, 634050 Tomsk, Russia \\ ${ }^{b}$ Tomsk Polytechnic University, 30 Lenin st., 634050 Tomsk, Russia \\ ${ }^{c}$ N. N. Vorozhtsov Novosibirsk Institute of Organic Chemistry, SB RAS, Acad. Lavrentjev Ave. 9, \\ Novosibirsk 630090, Russia \\ ${ }^{d}$ Department of Chemistry, University of Minnesota Duluth, Duluth, Minnesota 55812, USA \\ E-mail:vzhdanki@d.umn.edu
}

\section{Dedicated to Professor Nikolai Zefirov on his $70^{\text {th }}$ birthday}

(received 22 Mar 05; accepted 25 May 05; published on the web 27 May 05)

\begin{abstract}
Reactions of (dichloroiodo)benzene or (dichloroiodo)benzene/iodine with various monoterpenes in methanol provide a selective approach to the respective products of chloromethoxylation or iodomethoxylation of the double bond. The halomethoxylation of $(+)-3$-carene, carvone, and $\beta$ pinene proceeds with especially high regio- and stereoselectivity affording an appropriate single product in each case, while the reaction of limonene gives products of functionalization of both double bonds. This new methodology for the controlled introduction of halogen substituents into the structure of naturally occuring monoterpenes provides an entry into various potentially important synthetic intermediates.
\end{abstract}

Keywords: Monoterpenes, carene, carvone, pinene, limonene, (dichloroiodo)benzene, chloromethoxylation, iodomethoxylation

\section{Introduction}

The controlled introduction of halogen substituents into the structure of naturally occuring monoterpenes provides an entry into a plethora of important synthetic intermediates and other practically useful products. ${ }^{1-4}$ Numerous examples of bromination, ${ }^{2}$ chlorination, ${ }^{3}$ and iodination ${ }^{4}$ of carvone, pinene, limonene, citral, camphen, pulegone and other terpenes and terpenoids have been reported in the literature. The most common and best investigated reaction is the bromination of terpenes using bromine, ${ }^{2 \mathrm{a}} \mathrm{N}$-bromosuccinimide, ${ }^{2 \mathrm{~b}}$ and other brominating reagents. ${ }^{2 c}$ In contrast, the chemoselective introduction of chlorine or iodine into a terpene 
structure represents a challenging problem. Several examples of a relatively selective chlorination of terpenoids with hypochlorous acid, ${ }^{3 \mathrm{a}}$ tert-butylhypochlorite, ${ }^{3 \mathrm{~b}}$ or nitrosyl chloride $^{3 c}$ were reported in the literature. Iodination of terpenes has previously been achieved by using iodine or $N$-iodosuccinimide. ${ }^{4}$

(Dichloroiodo)benzene is commonly used as an exceptionally selective chlorinating reagent. ${ }^{5}$ In particular, it was demonstrated that (dichloroiodo)benzene can be used for the chlorination of the $\alpha$-santonin derivatives under free-radical conditions. ${ }^{6}$ Recently, we reported that (dichloroiodo)benzene in methanol is a useful reagent for halomethoxylation of simple alkenes and alkynes. ${ }^{7}$ Specifically, we have found that (dichloroiodo)benzene or a combination of (dichloroiodo)benzene with iodine can serve as an efficient source of the electrophilic " $\mathrm{Cl}^{+}$" or "I " species, respectively, in the reactions with styrenes and arylacetylenes in the presence of methanol or water. ${ }^{7}$ In the present work, we extend the reaction of halomethoxylation of unsaturated compounds to the more complex system of natural monoterpenes.

\section{Results and Discussion}

We have investigated the reactions of (dichloroiodo)benzene or a combination (dichloroiodo)benzene/iodine in methanol with the following terpenes: (+)-3-carene (1a), carvone (1b), $\alpha$ - and $\beta$-pinenes (1c), and limonene (1d). Yields of products and reaction conditions are shown in Table 1.

The reaction of (+)-3-carene (1a) with $\mathrm{PhICl}_{2}$ in methanol at room temperature afforded product of anti-chloromethoxylation (2a) isolated by column chromatography in $42 \%$ yield. Under similar conditions, the reaction of carene (1a) with $\mathrm{PhICl}_{2} / \mathrm{I}_{2}$ resulted in a selective formation of a single diastereomer (3a) isolated in 90\% yield after column chromatography on silica gel (Scheme 1). The formation of single diastereomers (2a) and (3a) due to the Markovnikov-type anti-addition of the electrophilic reagent from the less sterically hindered side of the double bond in (1a) is in agreement with the typical reactivity of $(+)$-3-carene (for example, see bromoallenyloxylation of $(+)-3-$ carene $\left.^{8}\right)$. The structure of products $(\mathbf{2 a})$ and (3a) was assigned based on high resolution NMR and HRMS. In particular, in ${ }^{1} \mathrm{H}$ NMR of the iododerivative 3a, the vicinal coupling constants of $\mathrm{H}^{1}$ atom $\left({ }^{3} J_{\mathrm{H} 1-\mathrm{H} 2}=10.1\right.$ and $\left.4.4 \mathrm{~Hz}\right)$ and $\mathrm{H}^{6}$ atom $\left({ }^{3} J_{\mathrm{H} 6-\mathrm{H} 5}=8.3\right.$ and $\left.0.7 \mathrm{~Hz}\right)$ prove a half-chair conformation of the six-membered ring with atoms $\beta \mathrm{H}^{2}$ and $\alpha \mathrm{H}^{5}$ being pseudo-axial. Due to the pseudo-axial position, atom $\beta \mathrm{H}^{2}$ is shielded by the cyclopropane ring (up-field shift by $0.7 \mathrm{ppm}$ as compared to $\alpha \mathrm{H}^{2}$ ). Atom $\mathrm{H}^{4}$ in the iododerivative occupies an axial position $\left({ }^{3} J_{\mathrm{H} 4-\mathrm{H} 5}=11.6\right.$ and $\left.6.9 \mathrm{~Hz}\right)$ indicating $\alpha$-orientation of the iodine atom. Long-range spin-spin coupling ${ }^{4} J_{3 \mathrm{H} 10-\beta \mathrm{H} 2}=0.7 \mathrm{~Hz}$ is possible only in the case of the axial methyl trans to $\beta \mathrm{H}^{2}$, so the methoxy group is $\beta$-oriented. The configuration found (3- $\beta$ methoxy, 4- $\alpha$-iodo-) is in agreement with the typical asynchronous electrophilic trans-addition to 3-carene: primary attack of the electrophile $\left(\mathrm{I}^{+}\right)$from the less hindered $\alpha$-side of the $\mathrm{C}=\mathrm{C}$ bond of 
the planar six-membered cycle ${ }^{9}$ followed by addition of nucleophilic particle $\left(\mathrm{CH}_{3} \mathrm{OH}\right)$ from the opposite side.

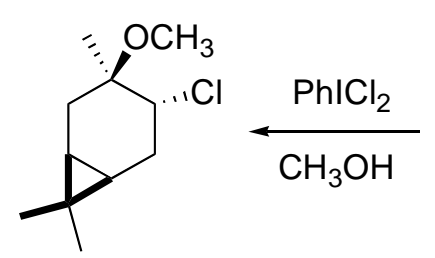

$2 a$

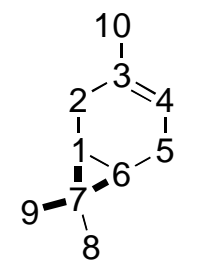

$1 \mathrm{a}$

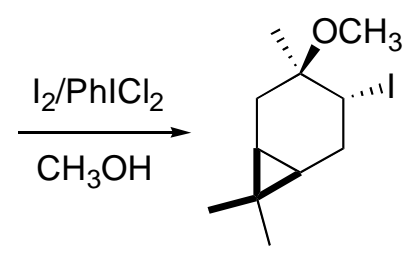

$3 a$

\section{Scheme 1}

In contrast to (+)-3-carene, the reaction of 2-carene with $\mathrm{PhICl}_{2} / \mathrm{I}_{2}$ resulted in the formation of a complex mixture of unstable products.

The reaction of carvone (1b) with $\mathrm{PhICl}_{2}$ or $\mathrm{PhICl}_{2} / \mathrm{I}_{2}$ in methanol selectively afforded the respective products of chloromethoxylation (2b) or iodomethoxylation (3b) of the terminal double bond, which were isolated by column chromatography in good yields (Scheme 2). It is interesting to note that the previously reported chlorination of $(+)$-carvone with hypochlorous $\operatorname{acid}^{10 \mathrm{a}}$ or the electrochemical chlorination ${ }^{10 \mathrm{~b}}$ of carvone led to a selective formation of the allylic chloride, 9-chlorocarvone. It was also reported that the bromination of carvone resulted in a nonselective addition to both double bonds and carbon C-6 affording the respective tetra- and pentabromide adducts. ${ }^{11}$

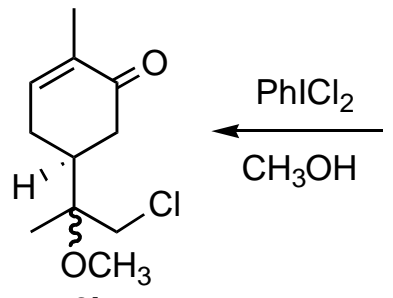

2b

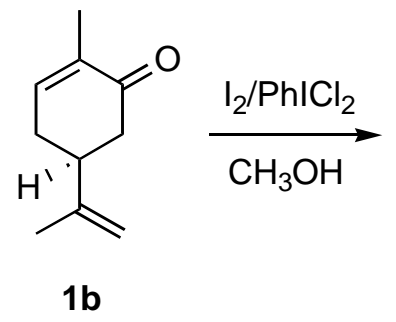

$1 b$<smiles>CC1=CC[C@@H](C(C)O)[C@H](CI)C1=O</smiles>

3b

\section{Scheme 2}

The reaction of $\alpha$-pinene and $\alpha$-terpineol with $\mathrm{PhICl}_{2}$ in methanol resulted only in the formation of a black tar, while a similar reaction of $\beta$-pinene (1c) afforded the product of ring opening (2c) (Scheme 3). Under conditions of iodomethoxylation, the reaction of $\beta$-pinene (1c) $\mathrm{PhICl}_{2} / \mathrm{I}_{2}$ in methanol afforded a 3:2 mixture of the expected iodide (3c) and the chloride (2c). Likewise, only the chloride (4c), instead of the expected iodide, was isolated from the reaction of $\beta$-pinene with $\mathrm{PhICl}_{2} / \mathrm{I}_{2}$ in aqueous acetonitrile (Scheme 4). 


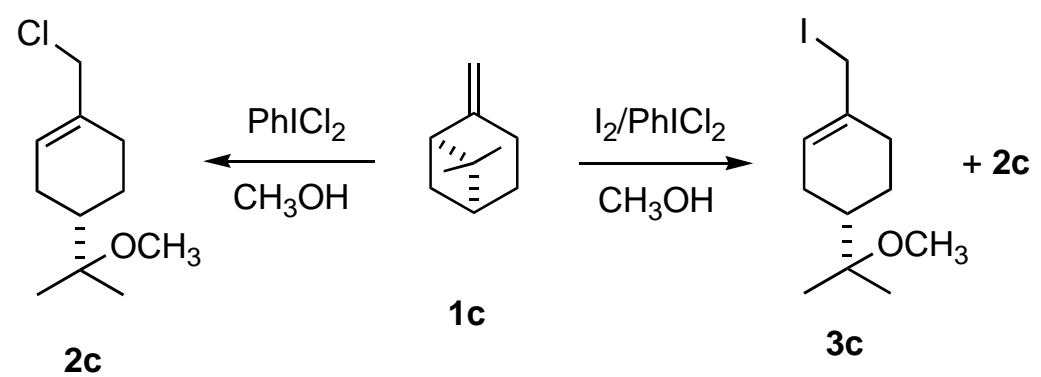

\section{Scheme 3}

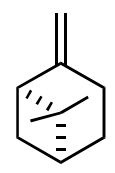

1c

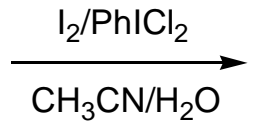

\section{Scheme 4}

A similar formation of the products of ring opening in the reaction of $\beta$-pinene with iodine ${ }^{11 a}$ and $\mathrm{NaOCl} / \mathrm{CeCl}_{3}{ }^{11 \mathrm{~b}}$ was previously reported in the literature. At the same time, the reaction of $\beta$-pinene with $N$-chlorosuccinimide in the presence of catalytic diphenyldiselenide $(3 \%)$ leads to the products of allylic chlorination with preserved pinene skeleton. ${ }^{11 \mathrm{c}}$

Only few examples of halogenation reactions of limonene (1d) were previously reported. ${ }^{13}$ In particular, it was found that bromine adds to both double bonds of limonene with the formation of the respective tetrabromide, ${ }^{13 a}$ while the reaction of limonene with tert-butyl hypochlorite leads to a complex mixture of mono- and dichlorides due to the allylic chlorination or electrophilic addition to the internal double bond. ${ }^{13 \mathrm{~b}}$

We have found that the chloromethoxylation reaction of limonene with $\mathrm{PhICl}_{2}$ in methanol has a low selectivity and leads to a large number of products, which we were not able isolate and identify. The iodomethoxylation of limonene with $\mathrm{PhICl}_{2} / \mathrm{I}_{2}$ in methanol was more selective and we were able to isolate two major chromatographic fractions from the reaction mixture: the first fraction containing regioisomers $\mathbf{3 d}$ and $\mathbf{6 d}$ and the second fraction - diastereomers $\mathbf{7 d}$ and $\mathbf{8 d}$ (Scheme 5). 
<smiles>C=C(C)C1CC=C(C)CC1</smiles>

1d

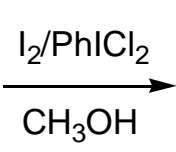

3d

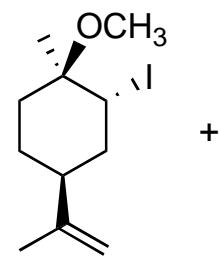

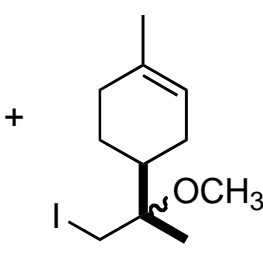

6d

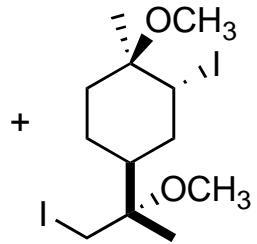

7d

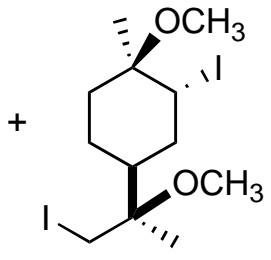

8d

\section{Scheme 5}

It should be emphasized that the reactions of iodomethoxylation in all cases were more selective compared to the chloromethoxylations. In particular, the GC-MS analysis of the reaction mixtures resulting from the chloromethoxylation of terpenes 1a-1c indicated the presence of polychlorinated compounds as by-products in these reactions. The formation of these polychlorinated by-products explains relatively lower preparative yields of the products of chloromethoxylation (see Table 1).

Table 1. Halomethoxylation of monoterpenes 1a-d using (dichloroiodo)benzene via Schemes 15

\begin{tabular}{cccccc}
\hline Entry & Monoterpene & Product & $\mathrm{Conditions}^{\mathrm{a}}$ & Time (min) & Yield $^{\mathrm{b}} \%$ \\
\hline 1 & $\mathbf{1 a}$ & $\mathbf{2 a}$ & $\mathrm{PhICl}_{2}, \mathrm{CH}_{3} \mathrm{OH}$ & 20 & 42 \\
2 & $\mathbf{1 a}$ & $\mathbf{3 a}$ & $\mathrm{I}_{2}, \mathrm{PhICl}_{2}, \mathrm{CH}_{3} \mathrm{OH}$ & 15 & 90 \\
3 & $\mathbf{1 b}$ & $\mathbf{2 b}$ & $\mathrm{PhICl}_{2}, \mathrm{CH}_{3} \mathrm{OH}$ & 20 & 43 \\
4 & $\mathbf{1 b}$ & $\mathbf{3 b}$ & $\mathrm{I}_{2}, \mathrm{PhICl}_{2}, \mathrm{CH}_{3} \mathrm{OH}$ & 15 & 62 \\
5 & $\mathbf{1 c}$ & $\mathbf{2 c}$ & $\mathrm{PhICl}_{2}, \mathrm{CH}_{3} \mathrm{OH}$ & 20 & 35 \\
6 & $\mathbf{1 c}$ & $\mathbf{2 c}$ & $\mathrm{I}_{2}, \mathrm{PhICl}_{2}, \mathrm{CH}_{3} \mathrm{OH}$ & 15 & 35 \\
& & $\mathbf{3 c}$ & & & 23 \\
7 & $\mathbf{1 c}$ & $\mathbf{4 c}$ & $\mathrm{I}_{2}, \mathrm{PhICl}_{2}, \mathrm{CH}_{3} \mathrm{CN}, \mathrm{H}_{2} \mathrm{O}$ & 15 & 21 \\
8 & $\mathbf{1 d}$ & $\mathbf{3 d}+\mathbf{6 d}$ & $\mathrm{I}_{2}, \mathrm{PhICl}_{2}, \mathrm{CH}_{3} \mathrm{OH}$ & 15 & 22 \\
& & $\mathbf{7 d}+\mathbf{8 d}$ & & & 13 \\
\hline
\end{tabular}

${ }^{a}$ All reactions were conducted at room temperature.

${ }^{\mathrm{b}}$ Preparative yields of products isolated after column chromatography.

In conclusion, the reactions of (dichloroiodo)benzene or (dichloroiodo)benzene/iodine with various monoterpenes in methanol provide a generally selective approach to the respective products of chloromethoxylation or iodomethoxylation of the double bond. The halomethoxylation of 3-carene, carvone, and $\beta$-pinene proceeds with especially high regio- and stereoselectivity affording an appropriate single product in each case, while the reaction of limonene gives products of functionalization of both double bonds. This new methodology for 
the controlled introduction of halogen substituents into the structure of naturally occuring monoterpenes provides an entry into various potentially important synthetic intermediates.

\section{Experimental Section}

General Procedures. IR spectra were recorded on a Bruker Vector-22 spectrophotometer. ${ }^{1} \mathrm{H}$ and ${ }^{13} \mathrm{C}$ NMR spectra were recorded on a Bruker AC-200 NMR spectrometer (200 and $50 \mathrm{MHz}$ ), AM-400 (400 and $100 \mathrm{MHz}$ ) and DRX 500 (500 and $125 \mathrm{MHz}$ ) using $\mathrm{Me}_{4} \mathrm{Si}$ as internal standard and $\mathrm{CDCl}_{3}$ or $\mathrm{CDCl}_{3}-\mathrm{CCl}_{4}$ 1:3. Chemical shifts are reported in parts per million (ppm). GC-MS spectra were obtained using Hewlett Packard 5890/II gas chromatograph equipped with quadrupole mass-spectrometer as detector (HP MSD 5971). High resolution EI mass spectra (EI, $70 \mathrm{eV}$ ) were recorded on Finnigan MAT 8200 mass spectrometer. Flash column chromatography was performed on silica gel L40/100 $\mu \mathrm{m}$ from Chemapol. Thin layer chromatography was carried out using TLC plates Sorbfil with fixed $\mathrm{SiO}_{2}$ layer. TLC plates were developed by spraying with the ethanol solution of vanillin $\left(2 \mathrm{~g}\right.$ vanillin and $5 \mathrm{ml}$ concentrated $\mathrm{H}_{2} \mathrm{SO}_{4}$ in $150 \mathrm{ml} \mathrm{EtOH})$ or $\mathrm{FeCl}_{3}\left(10 \%\right.$ solution of $\mathrm{FeCl}_{3}$ in $\left.\mathrm{EtOH}\right)$ followed by heating. All solvents were distilled before use. Pinenes were purchased from Fluka; limonene, carene and carvone were purchased from Aldrich. (Dichloroiodo)benzene was prepared by chlorination of iodobenzene with chlorine gas.

\section{General procedure for chloromethoxylation of monoterpenes 1a-d with (dichloroiodo)- benzene in methanol}

(Dichloroiodo)benzene $(0.281 \mathrm{~g}, 1.02 \mathrm{mmol})$ was added to a solution of monoterpene $(1.0 \mathrm{mmol})$ in $\mathrm{CH}_{3} \mathrm{OH}(3.0 \mathrm{ml})$ and the mixture was stirred for $20 \mathrm{~min}$ at room temperature. The resulting mixture was poured into water $(30 \mathrm{ml})$, extracted by ether $(2 \times 30 \mathrm{ml})$, washed with $5 \%-\mathrm{NaHCO}_{3}$ $(15 \mathrm{ml})$, water $(2 \times 50 \mathrm{ml})$, and saturated solution of $\mathrm{NaCl}(50 \mathrm{ml})$, and dried with $\mathrm{Na}_{2} \mathrm{SO}_{4}$. Ether was evaporated and the residue was dissolved in hexane $(3 \mathrm{ml})$ and separated by column chromatography using hexane first to isolate iodobenzene and then 5:1 mixture hexane-benzene to isolate reaction products $\mathbf{2 a}, \mathbf{2 b}$, and $\mathbf{2 c}$.

\section{General procedure for iodomethoxylation of monoterpenes 1a-d with (dichloroiodo)benzene/iodine in methanol}

(Dichloroiodo)benzene $(0.275 \mathrm{~g}, 1.0 \mathrm{mmol})$ was added to a solution of iodine $(0.267 \mathrm{~g}$, $1.05 \mathrm{mmol})$ in $\mathrm{CH}_{3} \mathrm{OH}(7.0 \mathrm{ml})$ and the mixture was stirred for $5 \mathrm{~min}$ at room temperature. The resulting mixture was added to a solution of monoterpene $(2.0 \mathrm{mmol})$ in $\mathrm{CH}_{3} \mathrm{OH}(1.0 \mathrm{ml})$ and the mixture was stirred for $10 \mathrm{~min}$ at room temperature. The resulting mixture was poured into 5\% aq. $\mathrm{Na}_{2} \mathrm{~S}_{2} \mathrm{O}_{3}(20 \mathrm{ml})$ and treated as described in the previous procedure. Column chromatography using hexane first and then 5:1 mixture hexane-benzene afforded products $\mathbf{3 a}, \mathbf{3 b}, \mathbf{3 c}, \mathbf{3 d}, \mathbf{6 d}, \mathbf{7 d}$ and $\mathbf{8 d}$. 


\section{Compound characterization}

$(1 S, 3 R, 4 R, 6 R)$-4-Chloro-3-methoxy-3,7,7-trimethylbicyclo[4.1.0]heptane (2a). oil; IR, $v_{\max }$, (neat, $\left.\mathrm{cm}^{-1}\right): 1115\left(\mathrm{C}-\mathrm{O}-\mathrm{CH}_{3}\right), 657(\mathrm{C}-\mathrm{Cl}) ;{ }^{1} \mathrm{H}$ NMR $\left(400 \mathrm{MHz}, \mathrm{CDCl}_{3}\right) \delta 0.67(1 \mathrm{H}$, ddd, H-6, J $\left.=9.9, \mathrm{~J}_{2}=8.3, \mathrm{~J}_{3}=0.7 \mathrm{~Hz}\right), 0.71\left(1 \mathrm{H}, \mathrm{ddd}, \mathrm{H}-1, \mathrm{~J}_{1}=10.1, \mathrm{~J}_{2}=9.9, \mathrm{~J}_{3}=4.4 \mathrm{~Hz}\right), 1.00$ and 1.02 $(3 \mathrm{H}, \mathrm{s}, \mathrm{H}-8$ and/or $3 \mathrm{H}, \mathrm{s}, \mathrm{H}-9), 1.27(3 \mathrm{H}, \mathrm{d}, \mathrm{H}-10, \mathrm{~J}=0.7 \mathrm{~Hz}), 1.48\left(1 \mathrm{H}, \mathrm{ddq}, \mathrm{H}-2 \beta, \mathrm{J}_{1}=14.2, \mathrm{~J}_{2}\right.$ $\left.=4.4, \mathrm{~J}_{3}=0.7 \mathrm{~Hz}\right), 2.00\left(1 \mathrm{H}, \mathrm{dd}, \mathrm{H}-2 \alpha, \mathrm{J}_{1}=14.2, \mathrm{~J}_{2}=10.1 \mathrm{~Hz}\right) ; 2.10\left(1 \mathrm{H}, \mathrm{ddd}, \mathrm{H}-5 \beta, \mathrm{J}_{1}=15.0\right.$, $\left.\mathrm{J}_{2}=6.9, \mathrm{~J}_{3}=0.7 \mathrm{~Hz}\right) ; 2.25\left(1 \mathrm{H}, \mathrm{ddd}, \mathrm{H}-5 \alpha, \mathrm{J}_{1}=15.0, \mathrm{~J}_{2}=11.6, \mathrm{~J}_{3}=8.3 \mathrm{~Hz}\right), 3.22\left(3 \mathrm{H}, \mathrm{s}, \mathrm{OCH}_{3}\right)$, $3.82\left(1 \mathrm{H}, \mathrm{dd}, \mathrm{H}-4, \mathrm{~J}_{1}=11.6, \mathrm{~J}_{2}=6.9 \mathrm{~Hz}\right) ;{ }^{13} \mathrm{C} \mathrm{NMR}\left(100 \mathrm{MHz}, \mathrm{CDCl}_{3}\right) \delta 15.53(\mathrm{C}-8), 16.61(\mathrm{C}-$ 1), 17.75 (C-7), 19.42 (C-6), 20.73 (C-10), 28.59 (C-9), 29.16 (C-5), 30.41 (C-2), $48.73\left(\mathrm{OCH}_{3}\right)$, 64.13 (C-4), 75.44 (C-3); HRMS: calcd for $\mathrm{C}_{11} \mathrm{H}_{19} \mathrm{ClO}\left(\mathrm{M}^{+}\right)$: 202.11243, found $\mathrm{m} / \mathrm{z} 202.11158$; MS (EI, $70 \mathrm{eV}$ ) m/z (\%): 202/204 (M+, 3), 187/189 (6), 170/172 (12), 149 (38), 135 (100), 119 (22), 106 (53), 93 (59), 85 (39), 73 (25), 55 (21), 43 (30).

$(1 S, 3 R, 4 R, 6 R)$-4-Iodo-3-methoxy-3,7,7-trimethylbicyclo[4.1.0]heptane (3a). oil; IR, $v_{\max }$, (neat, $\left.\mathrm{cm}^{-1}\right): 1107\left(\mathrm{C}-\mathrm{O}-\mathrm{CH}_{3}\right), 599(\mathrm{C}-\mathrm{I}) ;{ }^{1} \mathrm{H}$ NMR $\left(500 \mathrm{MHz}, \mathrm{CDCl}_{3}\right) \delta 0.45\left(1 \mathrm{H}\right.$, ddd, H-6, $J_{1}=$ $\left.9.9, J_{2}=8.3, J_{3}=0.7 \mathrm{~Hz}\right), 0.71\left(1 \mathrm{H}, \mathrm{ddd}, \mathrm{H}-1, J_{1}=10.1, J_{2}=9.9, J_{3}=4.4 \mathrm{~Hz}\right), 0.86$ and 0.93 $(3 \mathrm{H}, \mathrm{s}, \mathrm{H}-8$ and/or $3 \mathrm{H}, \mathrm{s}, \mathrm{H}-9), 1.19(3 \mathrm{H}, \mathrm{d}, \mathrm{H}-10, J=0.7 \mathrm{~Hz}) ; 1.31\left(1 \mathrm{H}, \mathrm{ddq}, \mathrm{H}-2 \beta, J_{1}=14.2, J_{2}\right.$ $\left.=4.4, J_{3}=0.7 \mathrm{~Hz}\right), 2.01\left(1 \mathrm{H}, \mathrm{dd}, \mathrm{H}-2 \alpha, J_{1}=14.2, J_{2}=10.1 \mathrm{~Hz}\right) ; 2.37\left(1 \mathrm{H}, \mathrm{ddd}, \mathrm{H}-5 \beta, J_{1}=15.0\right.$, $\left.J_{2}=6.9, J_{3}=0.7 \mathrm{~Hz}\right), 2.54\left(1 \mathrm{H}, \mathrm{H}-5 \alpha, J_{1}=15.0, J_{2}=11.6, J_{3}=8.3 \mathrm{~Hz}\right), 3.06\left(3 \mathrm{H}, \mathrm{s}, \mathrm{OCH}_{3}\right)$; $3.98\left(1 \mathrm{H}, \mathrm{dd}, \mathrm{H}-4, J_{1}=11.6, J_{2}=6.9 \mathrm{~Hz}\right) ;{ }^{13} \mathrm{C} \mathrm{NMR}\left(125 \mathrm{MHz}, \mathrm{CDCl}_{3}\right) \delta 15.10(\mathrm{C}-8), 17.45(\mathrm{C}-$ 7), 19.56 and 19.62 (C-1 and/or C-6), 21.98 (C-10), 26.90 (C-5), 28.43 (C-9), 33.86 (C-2), 41.08 (C-4), $48.28\left(\mathrm{OCH}_{3}\right), 73.88$ (C-3); HRMS: calcd for $\mathrm{C}_{11} \mathrm{H}_{18} \mathrm{O}(\mathrm{M}-\mathrm{HI})^{+}: 166.13576$, found $\mathrm{m} / \mathrm{z}$ 166.13244; MS (EI, $70 \mathrm{eV}) \mathrm{m} / \mathrm{z}(\%): 294\left(\mathrm{M}^{+},<1\right), 167$ (6), 166 (19), 151 (22), 142 (41), 135 (26), 106 (53), 119 (44), 93 (100), 85 (39), 67 (33), 59 (23), 55 (24), 43 (68).

5-(1-Chloro-2-methoxypropan-2-yl)-2-methylcyclohex-2-enone (2b), mixture of epimers (2:1). oil; IR, $v_{\max }$, (neat), $\mathrm{cm}^{-1}$ : $1724(\mathrm{C}=\mathrm{C}), 1675(\mathrm{C}=\mathrm{O}), 1257\left(\mathrm{CH}_{2}-\mathrm{Cl}\right), 1039\left(\mathrm{C}-\mathrm{O}-\mathrm{CH}_{3}\right), 751$ (C-Cl); ${ }^{1} \mathrm{H}$ NMR $\left(200 \mathrm{MHz}, \mathrm{CDCl}_{3}\right) \delta 1.12(3 \mathrm{H}, \mathrm{s}, \mathrm{H}-9) ; 1.70(3 \mathrm{H}, \mathrm{s}, \mathrm{H}-10) ; 2.05-2.5(5 \mathrm{H}, \mathrm{m}, \mathrm{H}-$ 4, H-5, H-6); 3.18 (3H, s, $\left.\mathrm{OCH}_{3}\right) ; 3.29-3.46(2 \mathrm{H}, \mathrm{m}, \mathrm{H}-8) ; 6.63\left(0.65 \mathrm{H}, \mathrm{m}_{1} \mathrm{~W}_{1 / 2}=10 \mathrm{~Hz}\right), 6.67$ $\left(0.35 \mathrm{H}, \mathrm{m}_{1} \mathrm{~W}_{1 / 2}=10 \mathrm{~Hz}\right) ;{ }^{13} \mathrm{C} \mathrm{NMR}\left(50 \mathrm{MHz}, \mathrm{CDCl}_{3}\right) \delta$ (main isomer) $15.79(\mathrm{C}-10), 18.07(\mathrm{C}-9)$, 27.20 (C-4), 38.64 (C-6), 41.10 (C-5), 47.89 (C-8), $49.97\left(\mathrm{OCH}_{3}\right), 77.22$ (C-7), 136.02 (C-2), 143.18 (C-3), 198.34 (C-1); (minor isomer) 15.79 (C-10), 17.99 (C-9), 26.46 (C-4), 39.24 (C-6), 41.10 (C-5), 47.25 (C-8), $50.09\left(\mathrm{OCH}_{3}\right) ; 77.86$ (C-7), 135.74 (C-2), 144.03 (C-3), 198.34 (C-1); HRMS: calcd for $\mathrm{C}_{11} \mathrm{H}_{17} \mathrm{ClO}_{2}\left(\mathrm{M}^{+}\right)$: 216.09170, found $\mathrm{m} / \mathrm{z} 216.09215$; MS (EI, $\left.70 \mathrm{eV}\right) \mathrm{m} / \mathrm{z}(\%)$ : 216/218 (M+, <1), 184/186 (3), 167 (15), 149 (2), 135 (17), 121 (4), 110 (56), 109 (66), 108 (34), 107 (100), 95 (11), 93 (9), 82 (9), 79 (13), 73 (29), 57 (42), 53 (10), 43 (10), 41 (14).

5-(1-Iodo-2-methoxypropan-2-yl)-2-methylcyclohex-2-enone (3b), mixture of epimers (5:4). oil; IR, $v_{\max }$, (neat), $\mathrm{cm}^{-1}: 1720(\mathrm{C}=\mathrm{C}), 1672(\mathrm{C}=\mathrm{O}), 1076\left(\mathrm{C}-\mathrm{O}-\mathrm{CH}_{3}\right), 614(\mathrm{C}-\mathrm{I}) ;{ }^{1} \mathrm{H}$ NMR (500 $\left.\mathrm{MHz}, \mathrm{CDCl}_{3}\right) \delta$ (main isomer) $1.25(3 \mathrm{H}, \mathrm{s}, \mathrm{H}-9), 1.73(3 \mathrm{H}, \mathrm{s}, \mathrm{H}-10), 2.12-2.50(5 \mathrm{H}, \mathrm{m}, \mathrm{H}-4, \mathrm{H}-5$, H-6), $3.18\left(3 \mathrm{H}, \mathrm{s}, \mathrm{OCH}_{3}\right), 3.21-3.29(2 \mathrm{H}, \mathrm{s}, \mathrm{H}-8), 6.68\left(1 \mathrm{H}, \mathrm{m}_{1} \mathrm{~W}_{1 / 2}=10 \mathrm{~Hz}, \mathrm{H}-2\right) ; \delta$ (minor isomer) $1.26(3 \mathrm{H}, \mathrm{s}, \mathrm{H}-9), 1.73(3 \mathrm{H}, \mathrm{s}, \mathrm{H}-10), 2.12-2.50(5 \mathrm{H}, \mathrm{m}, \mathrm{H}-4, \mathrm{H}-5, \mathrm{H}-6), 3.17(3 \mathrm{H}, \mathrm{s}$, $\left.\mathrm{OCH}_{3}\right), 3.21-3.29(2 \mathrm{H}, \mathrm{m}, \mathrm{H}-8), 6.63\left(1 \mathrm{H}, \mathrm{m}_{1} \mathrm{~W}_{1 / 2}=10 \mathrm{~Hz}, \mathrm{H}-2\right) ;{ }^{13} \mathrm{C} \mathrm{NMR}\left(125 \mathrm{MHz}, \mathrm{CDCl}_{3}\right) \delta$ 
(main isomer) 12.22 (C-8), 15.82 (C-10), 18.82 (C-9), 26.25 (C-4), 39.08 (C-6), 41.92 (C-5), $49.31\left(\mathrm{OCH}_{3}\right), 75.17(\mathrm{C}-7), 135.42(\mathrm{C}-2), 142.99$ (C-3), 198.16 (C-1); $\delta$ (minor isomer) 12.32 (C-8), 15.82 (C-10), 18.95 (C-9), 27.02 (C-4), 38.43 (C-6), 42.05 (C-5), $49.47\left(\mathrm{OCH}_{3}\right)$; 74.97 (C7), 135.78 (C-2), 144.11 (C-3), 198.36 (C-1); HRMS: calcd for $\mathrm{C}_{11} \mathrm{H}_{17} \mathrm{IO}_{2}\left(\mathrm{M}^{+}\right)$: 308.02751 , found m/z 308.02889; MS (EI, $70 \mathrm{eV}) \mathrm{m} / \mathrm{z}(\%): 308\left(\mathrm{M}^{+},<1\right), 199$ (86), 167 (9), 149 (14), 121 (8), 109 (21, 107 (36), 93 (10), 91 (10), 82 (11), 81 (17), 73 (43), 72 (100), 59 (12), 55 (12), 43 (20), 41 (33).

1-(Chloromethyl)-4-(2-methoxypropan-2-yl)cyclohex-1-ene (2c). oil; IR, $v_{\max }$, (neat), $\mathrm{cm}^{-1}$ : $1259\left(\mathrm{CH}_{2}-\mathrm{Cl}\right), 1079\left(\mathrm{C}-\mathrm{O}-\mathrm{CH}_{3}\right), 682(\mathrm{C}-\mathrm{Cl}) ;{ }^{1} \mathrm{H} \mathrm{NMR}\left(200 \mathrm{MHz}, \mathrm{CDCl}_{3}\right) \delta 1.08$ and $1.09(3 \mathrm{H}$, s, H-9 and/or 3H, s, H-10), $1.20-2.15$ (7H, m, H-3, H-4, H-5, H-6), 3.14 (3H, s, OCH $), 3.94$ $(2 \mathrm{H}, \mathrm{s}, \mathrm{H}-7), 5.77\left(1 \mathrm{H}, \mathrm{m}_{1} \mathrm{~W}_{1 / 2}=10 \mathrm{~Hz}, \mathrm{H}-2\right) ;{ }^{13} \mathrm{C} \mathrm{NMR}\left(50 \mathrm{MHz}, \mathrm{CDCl}_{3}\right) \delta 21.98$ and $22.21(\mathrm{C}-$ 9 and/or C-10), 23.50 (C-5), 26.81 and 27.04 (C-3 and/or C-6), 41.86 (C-4), $48.68\left(\mathrm{OCH}_{3}\right), 49.66$ (C-7), 76.11 (C-8), 127.16 (C-2), 134.45 (C-1); HRMS: calcd for $\mathrm{C}_{11} \mathrm{H}_{19} \mathrm{ClO}\left(\mathrm{M}^{+}\right)$: 202.11243, found m/z 202.11239; MS (EI, $70 \mathrm{eV}) \mathrm{m} / \mathrm{z}(\%):$ 202/204 (M+1 1), 170/172 (4), 166 (7), 135 (10), 134 (30), 123 (11), 119 (24), 105 (11), 93 (19), 91 (25), 84 (15), 73 (100), 55 (12), 43 (13).

2-(4-(Chloromethyl)cyclohex-3-enyl)propan-2-ol (4c). IR, $v_{\max }$, (neat), $\mathrm{cm}^{-1}: 3354(\mathrm{OH}), 1274$ $\left(\mathrm{CH}_{2}-\mathrm{Cl}\right), 1086(\mathrm{C}-\mathrm{OH}), 682(\mathrm{C}-\mathrm{Cl}) ;{ }^{1} \mathrm{H}$ NMR $\left(500 \mathrm{MHz}, \mathrm{CDCl}_{3}-\mathrm{CCl}_{4}-1: 3\right) \delta 1.10$ and 1.12 (3H, s, H-9 and/or 3H, s, H-10), 1.22 - 2.22 (7H, m, H-3, H-4, H-5, H-6), 3.90 (2H, s, H-7), 5.72 $\left(1 \mathrm{H}, \mathrm{m}_{1} \mathrm{~W}_{1 / 2}=10 \mathrm{~Hz}, \mathrm{H}-2\right) ;{ }^{13} \mathrm{C} \mathrm{NMR}\left(125 \mathrm{MHz}, \mathrm{CDCl}_{3} / \mathrm{CCl}_{4}-1: 3\right) \delta 23.46(\mathrm{C}-5), 25.59$ and 27.45 (C-9 and/or C-10), 26.79 and 26.86 (C-3 and/or C-6), 44.60 (C-4), 49.48 (C-7), 72.13 (C8), 126.77 (C-2), 134.30 (C-1); HRMS: calcd for $\mathrm{C}_{11} \mathrm{H}_{19} \mathrm{ClO}\left(\mathrm{M}^{+}\right)$: 188.09679, found $\mathrm{m} / \mathrm{z}$ 188.09653; MS (EI, 70 eV) m/z (\%): 188/190 (M+, <1), 187/189 (<1), 170/172 (14), 161/163 (11), 153 (100), 147/149 (10), 135 (15), 121 (19), 119 (21), 107 (23), 96 (50), 91 (23), 81 (91), 73 (32), 55 (19), 41 (23).

1-(Iodomethyl)-4-(2-methoxypropan-2-yl)cyclohex-1-ene (3c). oil; ${ }^{1} \mathrm{H}$ NMR (500 MHz, $\left.\mathrm{CDCl}_{3}\right) \delta 1.01$ and $1.03(3 \mathrm{H}, \mathrm{s}, \mathrm{H}-9$ and/or 3H, s, H-10), $1.18-2.18$ (7H, m, H-3, H-4, H-5, H6), $3.10\left(3 \mathrm{H}, \mathrm{s}, \mathrm{OCH}_{3}\right), 3.81(2 \mathrm{H}, \mathrm{s}, \mathrm{H}-7), 5.89\left(1 \mathrm{H}, \mathrm{m}_{1} \mathrm{~W}_{1 / 2}=10 \mathrm{~Hz}, \mathrm{H}-2\right) ;{ }^{13} \mathrm{C} \mathrm{NMR}(125 \mathrm{MHz}$, $\left.\mathrm{CDCl}_{3}\right) \delta 12.88(\mathrm{C}-7), 21.86$ and 22.07 (C-9 and/or C-10), 23.43 (C-5), 26.92 and 28.17 (C-3 and/or C-6), 41.75 (C-4), $48.55\left(\mathrm{OCH}_{3}\right), 49.66$ (C-7), 75.88 (C-8), 126.19 (C-2), 135.35 (C-1).

(1R,2R,4S)-2-Iodo-1-methoxy-1-methyl-4-(prop-1-en-2-yl)cyclohexane (3d). oil; ${ }^{1} \mathrm{H}$ NMR $\left(500 \mathrm{MHz}, \mathrm{CDCl}_{3}\right)$ ) $1.16(3 \mathrm{H}, \mathrm{s}, \mathrm{H}-10), 1.62(3 \mathrm{H}, \mathrm{s}, \mathrm{H}-9), 1.20-2.54$ (7H, m, H-3, H-4, H-5, $\mathrm{H}-6), 3.15\left(3 \mathrm{H}, \mathrm{s}, \mathrm{OCH}_{3}\right) ; 4.55$ (2H, s, H-2), 4.54 (1H, ddd, H-8, J=2.8, 2.8, $\left.2.8 \mathrm{~Hz}\right)$; ${ }^{13} \mathrm{C}$ NMR $\left(125 \mathrm{MHz}, \mathrm{CDCl}_{3}\right) \delta$ ) 17.91 (C-9), 23.42 (C-5), 23.52 (C-10), 33.63 and 36.97 (C-3, and/or C-6), 39.81 (C-4), 40.08 (C-2), $48.80\left(\mathrm{OCH}_{3}\right), 75.84$ (C-1), 109.50 (C-8), 148.43 (C-7); HRMS: calcd for $\mathrm{C}_{11} \mathrm{H}_{19} \mathrm{IO}\left(\mathrm{M}^{+}\right): 294.04810$, found $\mathrm{m} / \mathrm{z} 294.04824$.

4-(1-Iodo-2-methoxypropan-2-yl)-1-methylcyclohex-1-ene (6d), mixture of two isomers (1:1). oil; ${ }^{1} \mathrm{H}$ NMR (500 MHz, $\left.\mathrm{CDCl}_{3}\right) \delta$ (first isomer) $1.35(3 \mathrm{H}, \mathrm{s}, \mathrm{H}-9), 1.62(3 \mathrm{H}, \mathrm{s}, \mathrm{H}-10), 1.20$ -2.54 (7H, m, H-3, H-4, H-5, H-6), 3.17 (3H, s, OCH $)$; 3.22-3.36 (2H, m, H-8), $5.30\left(1 \mathrm{H}, \mathrm{m}_{1}\right.$ $\left.\mathrm{W}_{1 / 2}=10 \mathrm{~Hz}, \mathrm{H}-2\right) ; \delta$ (second isomer) $1.36(3 \mathrm{H}, \mathrm{s}, \mathrm{H}-9), 1.70(3 \mathrm{H}, \mathrm{s}, \mathrm{H}-10), 2.20-2.54(7 \mathrm{H}, \mathrm{m}$, H-3, H-4, H-5, H-6), $3.18\left(3 \mathrm{H}, \mathrm{s}, \mathrm{OCH}_{3}\right) ; 3.22-3.36(2 \mathrm{H}, \mathrm{m}, \mathrm{H}-8), 5.35\left(1 \mathrm{H}, \mathrm{m}_{1} \mathrm{~W}_{1 / 2}=10 \mathrm{~Hz}, \mathrm{H}-\right.$ 
2); ${ }^{13} \mathrm{C} \mathrm{NMR}\left(125 \mathrm{MHz}, \mathrm{CDCl}_{3}\right) \delta$ (first isomer) 14.63 (C-8), 21.32 and 25.99 (C-9 and/or C-10), 24.25 (C-5), 26.64 and 30.99 (C-3 and/or C-6), $48.95\left(\mathrm{OCH}_{3}\right), 75.74$ (C-7), 120.05 (C-2), 134.25 (C-1); $\delta$ (second isomer) 14.99 (C-8), 21.32 and 25.99 (C-9 and/or C-10), 25.73 (C-5), 26.31 and 30.86 (C-3 and/or C-6), $49.24\left(\mathrm{OCH}_{3}\right)$; 75.84 (C-7), 120.97 (C-2), 134.26 (C-1); HRMS: calcd for $\mathrm{C}_{11} \mathrm{H}_{19} \mathrm{IO}\left(\mathrm{M}^{+}\right)$: 294.04810, found $\mathrm{m} / \mathrm{z} 294.04824$.

2-Iodo-4-(2-iodo-1-methoxy-1-methyl-ethyl)-1-methoxy-1-methyl-cyclohexane (7d and 8d, mixture (1:1). oil; ${ }^{1} \mathrm{H}$ NMR $\left(500 \mathrm{MHz}, \mathrm{CDCl}_{3}\right) \delta$ (first isomer) $1.12(3 \mathrm{H}, \mathrm{s}, \mathrm{H}-9), 1.29(3 \mathrm{H}, \mathrm{s}, \mathrm{H}-$ 10), 2.18-2.24 (2H, m, H-3), 1.39-1.48 (2H, m, H-5), 1.62-170 (2H, m, H-6), 1.82-1.92 (1H, m, $\mathrm{H}-4), 3.12\left(3 \mathrm{H}, \mathrm{s}, \mathrm{OCH}_{3}\right), 4.52(1 \mathrm{H}, \mathrm{ddd} \mathrm{J}=2.8,2.8,2.8 \mathrm{~Hz}, \mathrm{H}-2) ; \delta$ (second isomer) $1.18(3 \mathrm{H}, \mathrm{s}$, H-9), 1.35 (3H, s, H-10), 2.18-2.24 (2H, m, H-3), 1.39-1.48 (2H, m, H-5), 1.62-170 (2H, m, H6), 1.82-1.92 (1H, m, H-4), $3.13\left(3 \mathrm{H}, \mathrm{s}, \mathrm{OCH}_{3}\right), 4.55(1 \mathrm{H}, \mathrm{ddd} \mathrm{J}=2.8,2.8,2.8 \mathrm{~Hz}, \mathrm{H}-2) ;{ }^{13} \mathrm{C}$ NMR (125 MHz, $\left.\mathrm{CDCl}_{3}\right) \delta$ (first isomer) 14.89 (C-8), 20.40 (C-10), 21.54 (C-5), 25.79 (C-9), 30.89 (C-5), 32.44 (C-6), 37.94 (C-2), 39.79 (C-4), 48.66 and $48.81\left(\mathrm{OCH}_{3}\right), 74.26(\mathrm{C}-1), 75.27$ (C-7); $\delta$ (second isomer) 15.16 (C-8), 20.57 (C-10), 22.11 (C-5), 25.79 (C-9), 31.23 (C-5), 32.67 (C-6), 38.44 (C-2), 39.96 (C-4), 49.12 and $49.19\left(\mathrm{OCH}_{3}\right), 74.35$ (C-1), 75.35 (C-7).

\section{Acknowledgements}

MSY thanks Ministry of Education (grant N E02-5.0-176) and RFFI (grant N 05-03-32582-a) for the financial support of this work. VVZ is thankful to the National Science Foundation (NSF/CHE-0353541) for partial support of this research.

\section{References and Footnotes}

1. Erman, W. F. Chemistry of the Monoterpenes: An Encyclopedic Handbook; M. Dekker: New York, 1985.

2. (a) Garcia, M.; Machado, F. W. L.; Maçaira, L. A.; Rabi, J. A. Tetrahedron Lett. 1980, 21, 777. (b) Bentley, P. D.; Cheetman, R.; Huff, R. K.; Swanbrough, J. Pestic. Sci. 1980, 11, 165. (c) Singh, N.; Verma, S. M. Indian J. Chem. 1982, 21, 680. (d) Antkowiak, R.; Antkowiak, W. Z. Tetrahedron Lett. 1994, 29, 5283. (e) Carman, R. M.; Shaw, I. M. Austral. J. Chem. 1980, 33, 1631. (f) Kurzer, F.; Patel, J. N. J. Org. Chem. 1988, 53, 258. (g) Ravindranath, B.; Srinivas, P. Indian J. Chem. 1983, 22, 592. (h) Tadahiro, K.; Ishii, K.; Ichinose, I.; Nakai, Y.; Kumagai, T. Chem. Commun. 1980, 1106.

3. (a) Hegde, S. G.; Vogel, M. K.; Saddler, J.; Hrinyo, T.; Rockwell, N.; Haynes, R.; Oliver, M.; Wolinsky, J. Tetrahedron Lett. 1980, 21, 441; (b) Hegde, S. G.; Wolinsky, J. Tetrahedron Lett. 1981, 22, 5019. (c) Ravindranath, B.; Srinivas P. Indian J. Chem. 1985, 24, 163. (d) Markova, Y. V.; Tkachev, A. V. Mendeleev Commun. 2000, 146. 
4. (a) Taneja, S. C.; Dhar, K. L.; Atal, C. K. J. Org. Chem. 1978, 43, 997. (b) Dondas, H. A.; Grigg, R.; Hadjisoteriou, M.; Markandu, J.; Kennewell, P.; Thornton-Pett, M. Tetrahedron 2001, 57, 1119. (c) Antonioletti, R.; Auria, M. D.; Piancatelli, G.; Scettri, A. Tetrahedron Lett. 1981, 22, 1041.

5. (a) Varvoglis, A. Hypervalent Iodine in Organic Synthesis; Academic Press: London, 1997.

(b) Varvoglis, A. The Organic Chemistry of Polycoordinated Iodine; VCH Publishers, Inc.: New York, 1992. (c) Zhdankin, V. V.; Stang, P. J. Chem. Rev. 2002, 102, 2523. (d) Stang, P. J.; Zhdankin, V. V. Chem. Rev. 1996, 96, 1123. (e) Zhdankin, V. V.; Stang, P. J. In Chemistry of Hypervalent Compounds; Akiba, K., Ed.; VCH Publishers: New York, 1999; Ch. 11, p 327.

6. Yasuo, F.; Hiroyuki, M.; Kazushige, N.; Takeshi, S.; Tatsuno Takastu, T. Heterocycles 1984, 22, 1477.

7. (a) Yusubov, M. S.; Yusubova, R. J.; Filimonov, V. D.; Chi, K.-W. Zh. Org. Khim. 2002, 38, 944. (b) Yusubov, M. S.; Yusubova, R. J.; Filimonov, V. D.; Chi, K.-W. Synth. Commun. 2004, 34, 443.

8. Dulcere, J.-P.; Crandall, J; Faure, R.; Santelli, M.; Agati, V.; Mihoubi, M. N. J. Org. Chem. 1993, 58, 5702.

9. Tkachev, A. V.; Denisov, A. V. Mendeleev Commun. 1991, 3, 98.

10. (a) Hegde, S. G.; Wolinsky, J. J. Org. Chem. 1982, 47, 3148. (b) Torii, S.; Uneyama, K.; Nakai, T.; Yasuda, T. Tetrahedron Lett. 1981, 22, 2291.

11. Carman, R. M.; Venzke, B. N. Austr. J. Chem. 1990, 43, 1909.

12. (a) Barton, D. H. R.; Blair, I. A.; Magnus, P. D. J. Chem. Soc., Perkin Trans. 2 1972, 614. (b) Moreno-Dorado, F. J.; Guerra, F. M.; Manzano, F. L.; Aladro, F. J.; Jorge, Z. D.; Massanet, G. M. Tetrahedron Lett. 2003, 44, 6691. (c) Hori, T.; Sharpless, K. B. J. Org. Chem. 1979, 44, 4208.

13. (a) Carman, R. M.; Smith, A. G.; Venzke, B. N. Austr. J. Chem. 1994, 47, 1387. (b) Ravindranath, B.; Srinivas, P. Indian J. Chem. (B) 1985, 24B, 163. 